\title{
EFFECT OF CYCLIC LED LIGHTING IN INCUBATOR ON HATCHING PERFORMANCE, CHICK QUALITY AND SEX RATIO OF BROILER CHICKS
}

\author{
M.I. El Sabry ${ }^{1}$ and H.G.M. Essa ${ }^{2}$ \\ 1-Animal Production Department, Faculty of Agriculture, Cairo University, 12613, Giza Egypt, 2-Alwatania \\ Poultry-Egypt, El Sadat City, Egypt
}

\section{SUMMARY}

Utilizing different colors of cool LED light during egg incubation process has shown positive effects on hatchability performance, however, the use of LED light still needs more investigation. This experiment was conducted to evaluate the effect of cyclical white LED light, during the first eighteen days of incubation period, on hatchability, chick quality and secondary sex ratio of broiler eggs. A total of 600 eggs from 34 wk old Cobb broiler breeders were divided into two groups, 300 eggs each. The treated group was incubated in 12-h light: $12 \mathrm{~h}$ dark (LD) regimen from day 0 to day 18 of incubation period, while the control group was incubated in complete darkness (DD). Egg weight loss \%, chick weight, hatching performance (total incubation period, embryonic mortalities, hatchability rate, and hatching window), chick quality (chick weight, Tona score, sellable chick \% and chick yield \%) and secondary sex ratio were determined.

Using cyclical white LED light showed a positive influence on some economic parameters. The eggs of the LD group had higher hatchability of fertile eggs $(91.05 \%, P=0.1)$ and sellable chicks $\%$ at hatch $(89.6 \%, P=$ 0.16) compared to those of DD treatment $(86.99 \%$ and $86.6 \%$, respectively). In addition, the total incubation period of eggs (hours) of the LD group (495.7 hours) was shorter $(P=0.01)$ than that (498.1 hours) of the DD group. However, the results show that egg weight loss \%, hatching window, chick weight and Tona score and secondary sex ratio of eggs exposed to LED light were similar to eggs hatched in darkness. The results indicate that providing cyclic LED light during incubation process improves hatchability and shorten the total incubation period with no deleterious effect on chick quality or significant changes in secondary sex ratio in broilers.

\section{Keywords: LED light; incubation; hatchability; broiler eggs}

\section{INTRODUCTION}

It is well known that incubation environment plays an important role in embryonic development. For example, temperature, humidity, ventilation, and egg turning can determine the success of incubation process. Fairchild and Christensen (2000) suggested light as a possible fifth environmental variable, which is not monitored during the incubation of avian eggs. The importance of light seems logical because under natural conditions, the hen leaves the nest periodically to feed and drink (Rogers, 1996), as well as the embryos can respond to light as early as $3 \mathrm{~d}$ of embryonic age (Erwin et al., 1971).

Shafey and Al-Mohsen (2002) and Hluchy et al. (2012) suggested that there are some factors which can impact the outcome of utilizing lighting in the incubators. These factors include: 1) source, color and intensity of light, 2) egg size and 3) eggshell characteristics. Moreover, both lighting hours and timing of light exposure are very important restrictions for the success of lighting during incubation. They have significant effects on the embryo's physiological traits, hatchability, chick quality and performance of post-hatch (Özkan et al., 2012 a,b; Archer and Mench. 2013; and Archer, 2015).

The contradictory reports about the influence of light on the embryonic mortality, hatchability rate and chick quality are probably the main reasons for ignoring it as an important environmental factor. For example, Archer (2015) found that there was no effect due to using light on the embryonic mortality, while Shafey and Al-Mohsen, (2002) found that light treatment significantly decreased early and late embryonic mortalities. In turkey, Kicka et al. (1982) and Fairchild and Christensen (2000) found that light treatment had no effect on hatchability rate, while Archer (2015) stated that hatchability rate was significantly increased due to light utilization (18 or 21 hours/ day) in the incubator during the first 18 of incubation or the entire 21 days of incubation.

Because of the conflict about the importance of light in avian egg incubation process, this study was conducted to investigate the effect of LED light on egg weight loss, chick weight, embryonic hatching performance, chick quality and secondary sex ratio.

\section{MATERIALS AND METHODS}

Six hundred eggs from $34 \mathrm{wk}$ old commercial Cobb flock were used in this experiment. Two identical incubators and one hatcher were used; the front windows were covered with black plastic sheet to prevent light intrusion into the incubator. One incubator was operated with the common procedure of incubation at complete darkness (DD), while the other one was outfitted with (white LED light strip) 
on each level, with one strip running the length of the racks. Each LED strip was attached to metal frame of the upper rack. The light was controlled by a timer, with a $12 \mathrm{hr} \mathrm{L:} 12 \mathrm{hrD}$ light schedule (LD) at 300 lux at egg level. Two replicates of 50 eggs each were set on each rack, for a total of six replicates over three levels equaling 300 eggs per incubator. Eggs of both treatments received standard temperature and humidity levels of $37.5^{\circ} \mathrm{C}$ and $52 \%$ relative humidity (RH). The eggs were incubated for $18 \mathrm{~d}$, and then they were moved into a hatcher. Each group was weighed at 0 time and re-weighed at $\mathrm{d} 18$ to calculate egg weight loss $\%=($ Initial egg weight at 0 time egg weight at $d 18$ )/ Initial egg weight at 0 time $* 100$. During the last 3 days, eggs were incubated in the same hatcher at $36.5^{\circ} \mathrm{C}$ and $65 \%$ RH. Hatching window was considered from $1 \%$ hatch to complete hatch max at 500hours of incubation time. All the chicks were weighed and counted within 45 min after hatch. The chick yield was calculated $=$ chick weight (g) / egg weight $(\mathrm{g}) * 100$.

Feather sexing was used for identification of males and females at one-day-old. In feather sexable broilers, slow- feathering chicks are male and fastfeathering chicks are female (Cobb-Vantress, 2008). The quality of the live chicks was assessed using Tona score, and they were categorized and counted as either sellable or cull chicks that are having any of the following: unhealed navel, leg abnormalities or too weak to stand, dirty or other abnormality. The unhatched eggs were broken out, the number of infertile, early death ( 0 to $7 \mathrm{~d}$ of incubation), middle death ( 8 to $14 \mathrm{~d}$ of incubation), and late death (15until hatch) eggs were recorded (Cobb-Vantress, 2008). Hatchability was calculated as a percentage of total eggs set, and was also calculated as a percentage of fertilized eggs. Sellable chicks were calculated as percentages of the total hatched chicks.
All of the assumptions of ANOVA were tested (Shapiro-Wilk test for normality). No transformations were needed to meet assumptions. All analyses were performed using JMP Pro 5 statistical analysis program. One-way ANOVA was used to investigate treatment effect on embryonic mortality, hatchability performance, chick quality traits and secondary sex ratio.

\section{RESULTS AND DISCUSSION}

\section{Embryonic mortality}

In the current study, the effects of white LED light from $\mathrm{d} 1$ to $\mathrm{d} 18$ of incubation on embryonic mortality, hatching performance, chick quality and secondary sex ratio in broiler breeder eggs were studied. This procedure could be applicable in commercial hatcheries. Results in Table (1) show that there were no significant differences between LD and DD treatments in the early, mid or late embryonic mortality percentage as well as percentage of pipped chicks. Our finding agrees with those of Huth and Archer (2015), who found that lighting (12L: 12D) the incubator and hatcher did not affect embryonic mortality, in layer hens and broiler breeder. Whilst, Shafey and Al-Mohsen, (2002) found that using 20watt green fluorescent light for $24 \mathrm{~h}$ during the first 18 days significantly decreased the embryonic mortality, while light did not affect the percentage of pipped with dead embryos.

Both egg weight loss \% and hatching window were not affected significantly by the LD light treatment (Table 2). These results may be due to similarity of the initial egg weight of the LD and DD groups were $59.7 \mathrm{~g}$ and $59 \mathrm{~g}$, respectively. No information is available about the effect of light on egg weight loss \% and hatching window.

Table 1. Comparison of embryonic mortality when eggs incubated in a 12:12 light cycle (LD) or 24-h darkness (DD)

\begin{tabular}{|c|c|c|c|c|}
\hline \multirow[b]{2}{*}{ Embryonic mortality (\%) } & \multicolumn{2}{|c|}{ Light treatment } & \multirow[b]{2}{*}{ p-Value } & \multirow[b]{2}{*}{ \pm SEM } \\
\hline & LD & DD & & \\
\hline Early & 4.32 & 7.84 & 0.07 & 1.26 \\
\hline Middle & 0.72 & 0.37 & 0.57 & 0.42 \\
\hline Late & 3.19 & 1.84 & 0.19 & 0.68 \\
\hline Pipped & 1.42 & 2.54 & 0.34 & 0.79 \\
\hline
\end{tabular}

No significant differences were found between different treatments

Table 2.Egg weight Loss \%, hatching window, total incubation period, chick weight and yield \% and Tona score for eggs incubated in a 12:12 light cycle (LD) or 24-h darkness (DD)

\begin{tabular}{lllll}
\hline & \multicolumn{3}{c}{ Light treatment } & \\
\cline { 2 - 4 } Parameters & LD & DD & p-Value & \pm SEM \\
\hline Egg weight loss (\%) & 12.31 & 12.11 & 0.72 & 0.38 \\
Hatching window (hours) & 23.37 & 23.41 & 0.96 & 0.57 \\
Total incubation period (hours) & $495.75^{\mathrm{b}}$ & $498.16^{\mathrm{a}}$ & 0.01 & 0.55 \\
Chick weight (g) & 42.63 & 42.35 & 0.43 & 0.24 \\
Chick Yield (\%) & 71.39 & 71.77 & 0.49 & 0.38 \\
Tona score & 88.66 & 88.33 & 0.89 & 1.7 \\
\hline
\end{tabular}

${ }^{\mathrm{a}, \mathrm{b}}$ Means, within a row, with different superscripts differ significantly. 
The length of incubation period of LD group was significantly shorter compared to that of the DD group (Table 2). These results may be due to the changes in the physiological and metabolic activities of the embryos due to exposure to light. Cooper et al. (2011) incubated the eggs of house sparrow (Passer domesticus) under different photoperiods similar to those found at temperate (18L: 6D) and tropical (12L: 12D) latitudes. The results of metabolic rate of embryos showed that the mean metabolic rate during the dark phase $\left(1.30 \pm 0.57 \mu \mathrm{L} \mathrm{CO}_{2} \mathrm{~min}^{-1} \mathrm{egg}^{-1}\right)$ was lower than that of light phase $\left(1.92 \pm 0.73 \mu \mathrm{L} \mathrm{CO}_{2} \mathrm{~min}^{-1} \mathrm{egg}^{-1}\right)$. In addition, Cooper et al. (2011) postulated that eggs incubated under the longer photoperiod (18 L: 8D) hatched about 1 day earlier than eggs incubated under the shorter photoperiod (12L:12 D). Also, Fairchild and Christensen (2000) found that the length of incubation period of turkey eggs was shortened by photo stimulation of eggs during the incubation process. The present results indicate that the light passes through the shell may play a key role in accelerating the embryos' development and metabolic activates in chicken. More studies should be done to understand how lighting can affect the pathway of metabolic activity.

Both chick weight $(\mathrm{g})$ and yield \% of both LD and DD treatments were similar (Table 2). These results are compatible with our egg weight loss \%, which were not affected by light treatment. These finding agree with those of Fairchild and Christensen (2000) in turkey. They found that chick weight was not affected by light treatment during incubation. While, Shafey and Al-Mohsen, (2002) found that chick weight $(\mathrm{g})$ and yield \% of the green light group were significantly lower than those of the dark group, in broiler breeders. This may be due to the different color light used in the experiments.

The differences between the overall hatchability, hatchability of fertile eggs and number of sellable chicks of LD and DD groups were not significant. However, it is noteworthy that the LD positively increased both overall hatchability percentage and hatchability of fertile eggs percentage by $5 \%$ as the most economic parameters that should be considered (Fig.1). These results are corresponded to findings by Hluchy et al. (2012), who tested different monochromatic lighting during incubation of broiler eggs and found that red light produced a higher hatchability than blue light, while the white light having the highest overall hatchability. In addition, Shafey (2004) found differences in hatchability among layer strains due to utilizing light during the incubation period. He suggested that the physical dimensions of eggs can allow different levels of light to pass through the eggshell. Archer et al. (2017) found a significant increase in the hatchability of chicken and Pekin duck eggs when they used a combination of white and red LED light during incubation. Moreover, our results partially agree with those of Shafey and Al-Mohsen, (2002), who found a significant increase in hatchability of fertile eggs percentage due to using green light throughout the incubation period. Huth and Archer (2015) found that both hatchability and percentage of chicks, with no defects, of the LED light group were significantly higher than those of un-treated group.

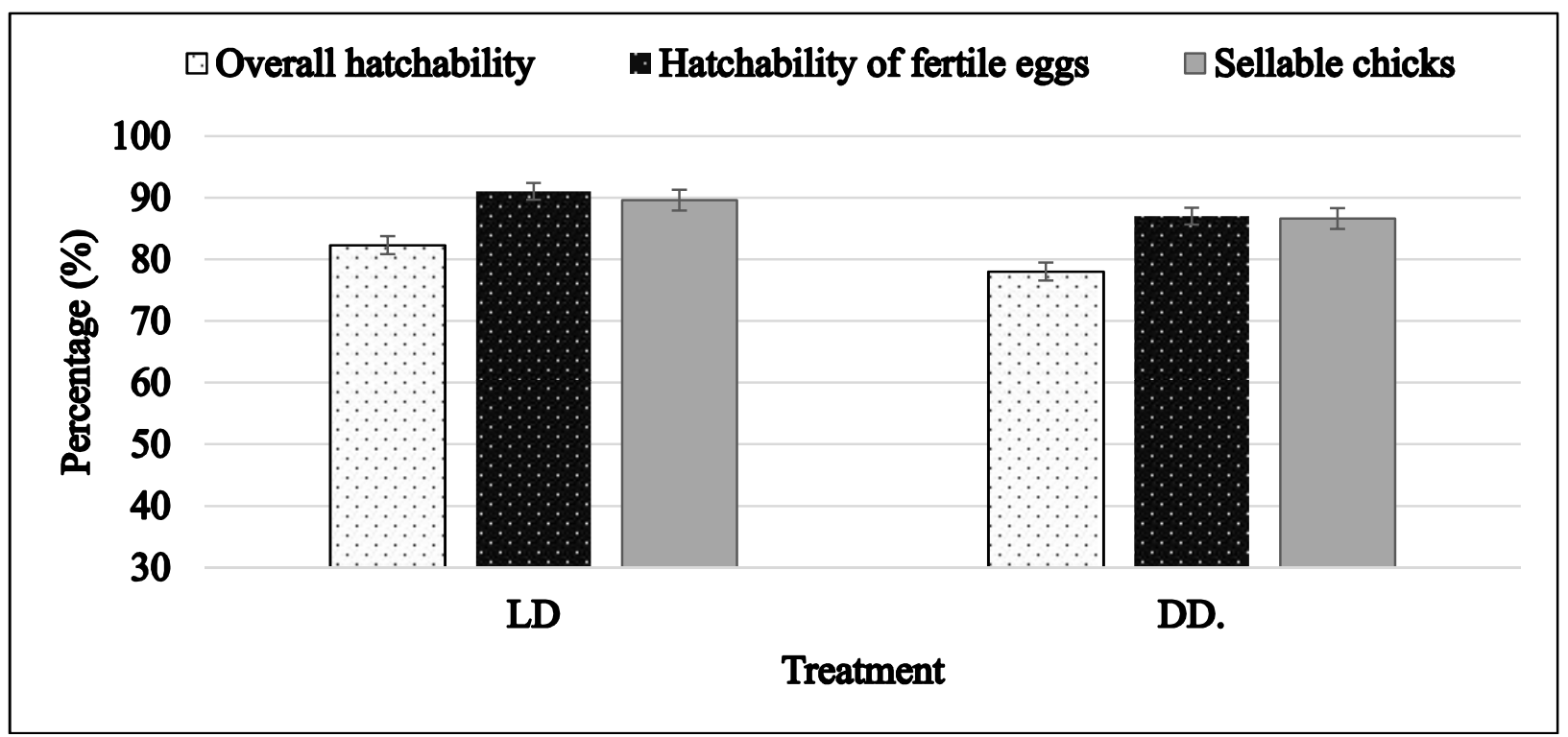

Fig.1. Comparison between overall hatchability, hatchability of fertile eggs and sellable chick of the treated (eggs incubated in a 12:12 light cycle, LD) or control group (24-h darkness, DD).

No significant differences were observed between the treated and control groups.

Tona score results show that there were no significant differences between LD and DD groups
(Table 2). On the other hand, previous studies, indicated that different light treatments during 
incubation improved chick quality compared to darkness control in turkeys (Fairchild and Christensen, 2000), and broilers (Archer et al., 2009; and Archer et al., 2017). The largest difference in the chick quality was attributed to unhealed navels, that the dark treatments having greater number of navel scores compared to the lighted treatments, in broiler breeders (Archer et al., 2017). This reduced un-healed navels percentage could be attributed to the faster growth rate of embryos of the light treated groups (Cooper et al., 2011).

Göth and Booth (2005) indicated that the incubation temperature can change the sex ratio. Since environmental variable can significantly affect sex ratio, it was anticipated that exposing eggs to white LED light during incubation process may influence the secondary sex ratio. In the present study, there were no significant differences in secondary sex ratio due to using light in the incubator. However, the female to male percent of the LD group was slightly greater than this of DD group (Fig. 2). The differential mortality might have been happening during the embryonic development due to the presence of light.

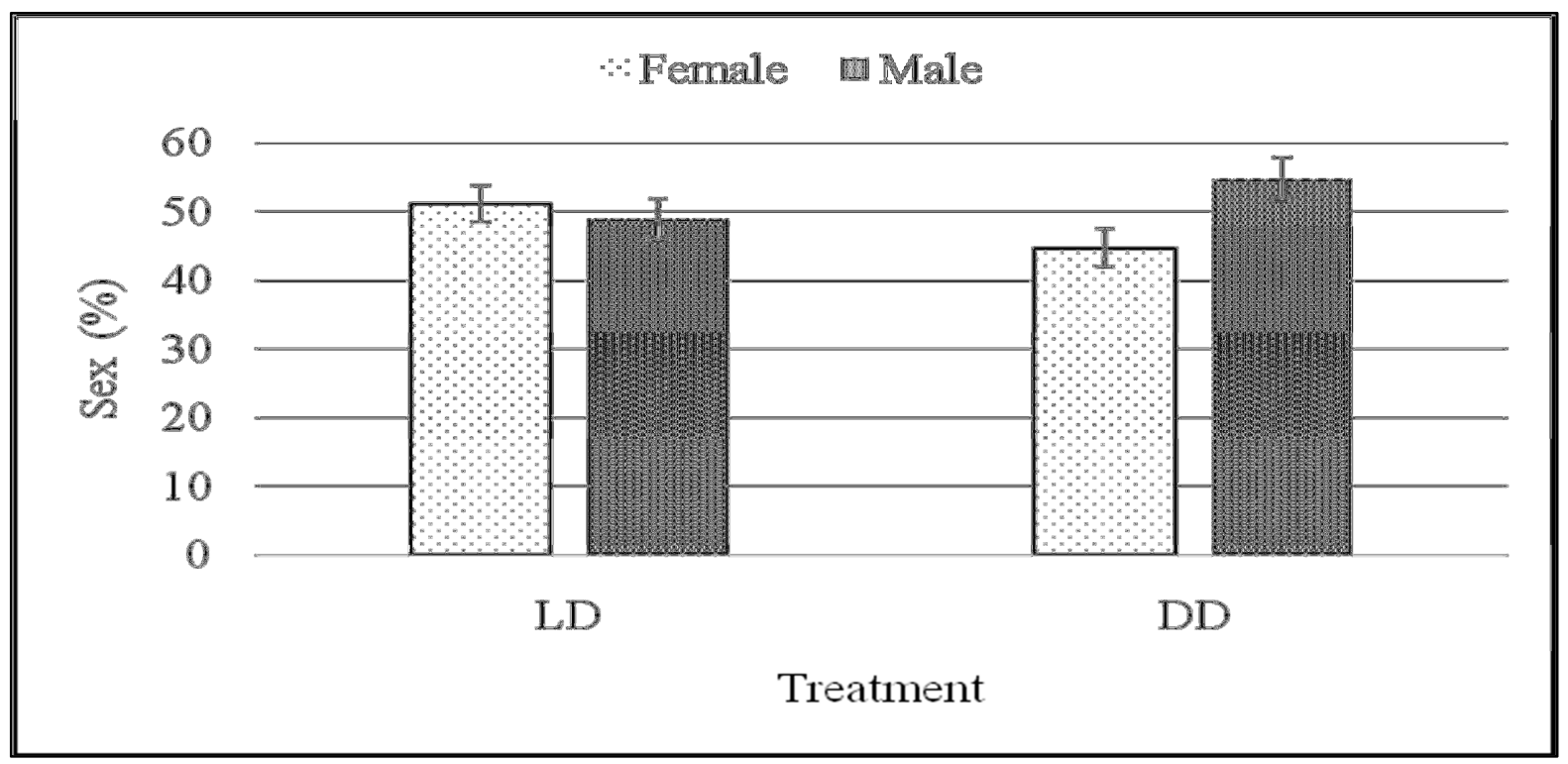

Fig.2. Comparison between secondary sex ratio of treated group (eggs incubated in a 12:12 light cycle, LD) or control group (24-h darkness, DD).

No significant differences were observed between the treated and control groups.

\section{CONCLUSION}

Providing cyclic white LED light during incubation of broiler eggs did slightly improve the measures of hatching and chick quality parameters. In addition, lighting did significantly shorten the incubation period. More benefits could be gained from this application after finding out the mechanism of light effects.

\section{REFERENCES}

Archer, G. S., D. Jeffrey and Z. Tucker, 2017. Effect of the combination of white and red LED lighting during incubation on layer, broiler, and Pekin duck hatchability. Poult. Sci. pex040.doi: $10.3382 / \mathrm{ps} / \mathrm{pex} 040$

Archer, G.S, 2015. Timing of light exposure during incubation to improve hatchability, chick quality and post-hatch well-being in broiler chickens: 21 or 18days. Int.J. Poult. Sci. 14(5):293-299.

Archer, G. S. and J. A. Mench, 2013. The effects of light stimulation during incubation on indicators of stress susceptibility in broilers. Poultry Science 92 :3103-3108

Archer, G. S., H. L. Shivaprasad, and J. A. Mench, 2009. Effect of providing light during incubation on the health, productivity, and behavior of broiler chickens. 88:29-37.

Cobb - Vantress, 2008. Cobb hatchery management guide.

http://cobbvantress.com/docs/defaultsource/guides/ cobb-hatchery-guide---english.pdf. Accessed 27 September 2016

Cooper, C.B., M.A. Voss, D.R. Ardia, S.H. Austin and W.D. Robinson, 2011.Light increases the rate of embryonic development: Implications for latitudinal trends in incubation period. Funct. Ecol., 25: 769-776.

Erwin, W. T., M. A. Boone, and B. D. Barnett, 1971. Response of developing embryo to light. Poult. Sci. 50:1883-1884.

Fairchild, B., and V. Christensen, 2000. Photo stimulation of turkey eggs accelerates hatching times without affecting hatchability, liver or heart growth, or glycogen content. Poult. Sci. 79:16271631. 
Göth A. and D.T. Booth, 2005. Temperaturedependent sex ratio in a bird. Biol. Lett. 31-33 doi:10.1098/rsbl.2004.0247

Hluchy, S., R. Toman, M. Cabaj and M. Adamkovicova, 2012. The effect of white and monochromatic lights on chicken hatching. Anim. Sci. Biotechnol., 45: 408-410.

Huth, J. C. and G. S. Archer, 2015.Effects of LED lighting during incubation on layer and broiler hatchability, chick quality, stress susceptibility and post-hatch growth. Poult Sci., 94 (12): 3052-3058. https://doi.org/10.3382/ps/pev298

Kicka, M. A., F.K.R. Stino, and G.A.R. Kamar, 1982. Influence of fluorescent light during incubation on hatch time and embryonic development of the chicken, turkey and duck egg. Arch. Gefluegelkd. 46:49-52.

Özkan, S., S. Yalçin, E. Babacanoğlu, H. Kozanoğlu, F. Karadaş, and S. Uysal. 2012. Photoperiodic lighting (16 hours of light: 8 hours of dark) programs during incubation: 1 . Effects on growth and circadian physiological traits of embryos and early stress response of broiler chickens. Poult. Sci. 91:2912-2921.
Özkan, S., S. Yalçin, E. Babacanoğlu, H. Kozanoğlu, F. Karadaş, and S. Uysal, 2012b. Photoperiodic lighting (16 hours of light:8 hours of dark) programs during incubation: 2. Effects on early post hatching growth, blood physiology, and production performance in broiler chickens in relation to post hatching lighting programs. Poult. Sci. 91:2922-2930.

Rogers, L. J, 1996. The Development of Brain and Behaviour in the Chicken. CABI Publishing, Wallingford, UK.

Shafey T. M. and T. H. Al-Mohsen, 2002. Embryonic Growth, Hatching Time and Hatchability Performance of Meat Breeder Eggs Incubated under Continuous Green Light. Asian-Aust. J. Anim. Sci. Vol 15, No. 12: 1702-1707.

Shafey, T, 2004. Effect of lighted incubation on embryonic growth and hatchability performance of two strains of layer breeder eggs. British Poultry Science 45:223 - 229 .

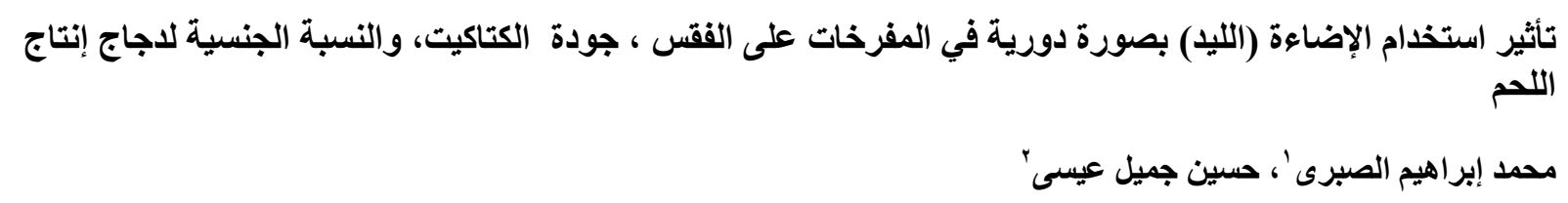

القسم الإنتاج الحيوانس، كلية الزراعة- جامعة القاهرة، + ـ الثركة الوطنية للدواجن (معدل التفريخ)- مدينة السادات، المنوفية، مصر

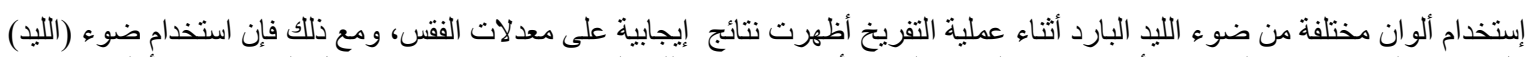

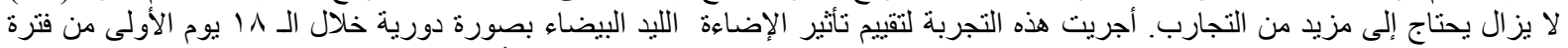

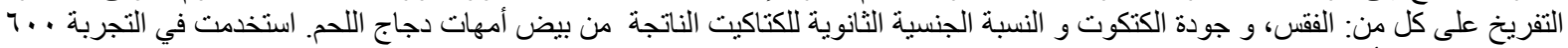

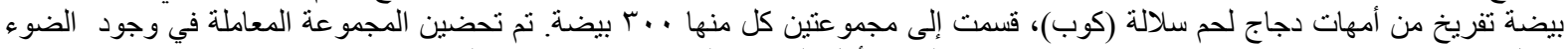

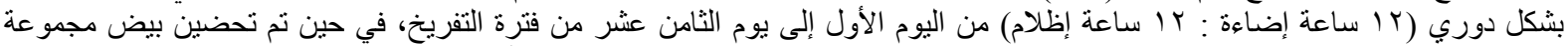

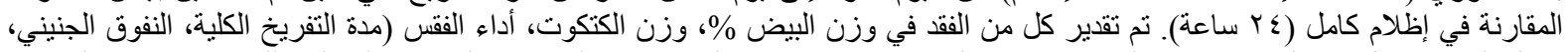

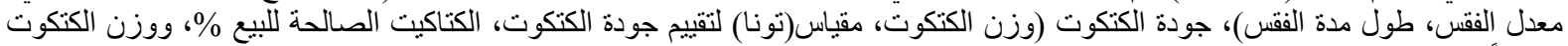

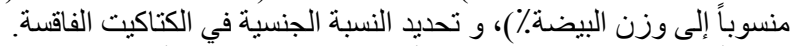

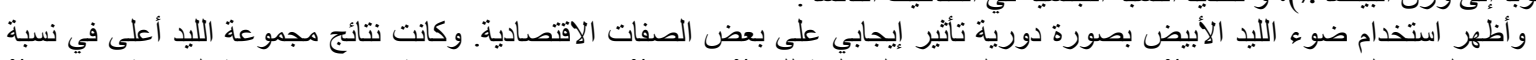

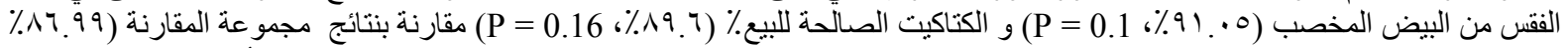

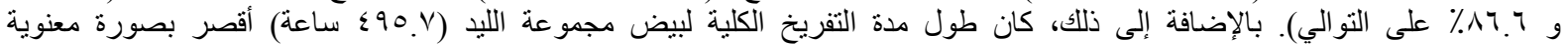

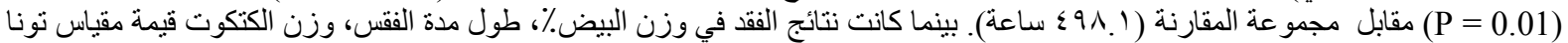

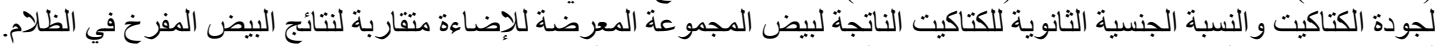

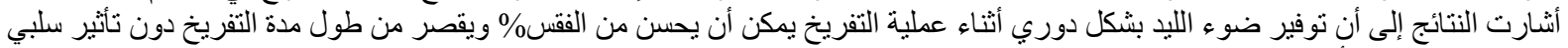
على جودة الكتكوت أو إحداث تغونير معنوي في النسبة الجنسية الثنانوية لكتاكيت إنتاج اللحم. 